\title{
PROPERTIES OF BLACK HOLES RELEVANT TO THEIR OBSERVATION*
}

\author{
JAMES M. BARDEEN \\ Yale University, U.S.A.
}

\begin{abstract}
Black holes are very small objects by astronomical standards, so in many circumstances they interact with their surroundings like a Newtonian mass point. However, if black holes are present in X-ray binary systems, the X-rays emitted in the inner part of the accretion disk probe the highly curved spacetime geometry near the horizon, particularly if the black hole is rapidly rotating. Some of the properties of circular orbits near the black hole are quite sensitive to the amount of angular momentum. The relativistic corrections remove a Newtonian degeneracy between several of the characteristic frequencies associated with perturbations of the circular orbits.

Hot spots in the inner part of the disk can produce dramatic fluctuations in intensity, since the frequency shifts of photons emitted by a given point on the disk are strongly time-dependent. The bending of the photon trajectories by the strong gravitational field can drastically affect the energy balance of the disk; much of the radiation emitted by the inner part of the disk is reabsorbed. The dragging of inertial frames by the angular momentum of the black hole can have striking consequences for the structure of the disk at quite large radii if the angular momentum of the accreting matter is not in the same direction as the angular momentum of the black hole.

Dynamic perturbations of black holes are now being intensively studied to see if there are any surprising physical effects associated with the rotation of the black hole. Unfortunately, though quite interesting methods of extracting energy from the black hole exist in principle most of them are unlikely to be realized to an important extent in the real astrophysical world.
\end{abstract}

\section{Introduction}

To a relativity theorist the most interesting observational aspects of black holes are those that reveal something of their intrinsic general relativistic nature. Ideally, one would like some observational confirmation of the Israel-Carter conjecture that the Kerr family of solutions to the Einstein equations includes all physically acceptable vacuum black hole geometries. The current indications that black holes probably do exist in certain X-ray binaries raise the hope that in the not-too-distant future we will be able to test strong field predictions of general relativity in a relatively clean way, without the uncertainties of the equation of state and radiation mechanisms that complicate interpretations of pulsar observations.

Therefore, I will concentrate in this lecture on the properties of black holes which strike me as most relevant to such strong field observations. Peebles (1972) has given an excellent review of how black holes might be detected through their long-range Newtonian gravitational field in globular clusters, galactic nuclei, etc. The detailed astrophysics of accretion into black holes will be reviewed at this Symposium by Sunyaev.

In order to observe strong-field effects some source of radiation must be present near, but outside the horizon of the black hole. The most likely source of the radiation is accreting matter flowing in some more or less continuous way into the black hole.

* Supported in part by the National Science Foundation (U.S.A.).

(: DeWitt-Morette led.), Gravitational Radiation and Gravitational Collapse, 132 144. All Rights Reserved. Copyright i 1974 by the $I A U$. 
Another, more remote possibility is a small, compact source of radiation (a 'star') orbiting near the black hole. Finally, the black hole may amplify an external wave disturbance or radiation may be emitted as the black hole settles down after being formed in gravitational collapse.

I assume that the metric describing the black hole is one of the Kerr metrics (Kerr, 1963). Theorems of Israel (1967), Carter (1971), and Hawking (Hawking and Ellis, 1973) establish almost conclusively that the Kerr metrics are the only stationary vacuum solutions of the Einstein equations which have regular event horizons and are asymptotically flat. The charged black holes, described by the Kerr-Newman metrics (Newman et al., 1965) are of little astrophysical interest. There are few mechanisms of charge separation in gravitational collapse capable of producing a significant charge on a black hole, and any initial charge will rather quickly be neutralized, since astrophysical plasmas are good conductors.

The Kerr metrics are a two-parameter family, characterized by gravitational mass $M$ and angular momentum $J$. Event horizons exist if and only if $M$ and $J$ satisfy $0 \leqslant c J / G M^{2} \leqslant 1$. It seems reasonable that centrifugal forces will prevent the complete collapse of any object with $a / M=c J / G M^{2}>1$; if a black hole is formed, some matter carrying the excess angular momentum must be left behind.

What values of the parameter $a / M$ can we reasonably expect for astrophysical black holes? This is an important question for the possibility of observing strong field effects. While if $a / M=1$ matter can exist in stable circular orbits arbitrarily close (in some senses) to the horizon, the radius of the innermost stable circular orbit increases rapidly as $a / M$ decreases from one, from $r / M=1$ when $a / M=1$, to $r / M \simeq 2$ when $a / M=0.95$, to $r / M \simeq 4$ when $a / M=0.5$, and to $r / M=6$ when $a / M=0$ (all in units with $G=c=1$ ). The ergosphere, where energy can be extracted from the black hole by emission of radiation or energetic particles in appropriate directions, extends from the horizon at $r=r_{+}=M+\left(M^{2}-a^{2}\right)^{1 / 2}$ to $r=2 M$ in the equatorial plane.

Calculations of Bardeen and Wagoner (1971) suggest that a black hole with $a / M$ infinitesimally close to one can be formed only by a quasi-stationary collapse which passes through a series of disk-like configurations. These disk-like configurations are probably unstable to a fragmentation process in which the central part of the disk, with $a / M<1$ by a finite amount, collapses dynamically. Thus, while many objects in the Galaxy have $c J / G M^{2} \gtrsim 1$ (the Sun, for instance), it seems unlikely that black holes will form with $a / M$ very close to one, though perhaps with $a / M$ as large as 0.9 or so.

Accretion of matter following the formation of the black hole can increase $a / M$. Bardeen (1970) has shown that an accretion process in which matter falls into the black hole from the innermost stable circular orbit in the equatorial plane can make $a / M=1$ after a finite amount of rest mass is accreted, provided that the binding energy released by the matter in getting into the innermost stable circular orbit all escapes to infinity. More realistic calculations, in which some radiation emitted by the accreting matter is captured by the black hole, suggest that accretion might be able to increase $a / M$ to a limiting value of perhaps 0.998 (Thorne, 1974). However, 
the rate of accretion cannot be so great that the radiation pressure of the energy released by the accreting matter exceeds the gravitational attraction of the black hole. The Eddington limit restricts the characteristic time scale for accretion to modify the black hole parameters to be greater than about $10^{8} \mathrm{yr}$. This sort of time scale is probably considerably longer than accretion can be maintained in a binary system composed of massive stars, but is not unreasonable for a large black hole in a galactic nucleus.

\section{Geodesics in the Kerr Metric}

Many observational aspects of black holes concern the properties of timelike and null geodesics. In the accretion disk models that have been suggested for galactic nuclei by Lynden-Bell (1969) and Lynden-Bell and Rees (1971) and elaborated for some of the X-ray binaries by Pringle and Rees (1972), Shakura and Sunyaev (1973), and Novikov and Thorne (1973) the matter to a good approximation follows circular geodesic orbits in the equatorial plane of the black hole. Such accretion disks seem to offer the best chance for tests of black hole theory in the near future.

Spherical accretion, with the black hole at rest in the interstellar medium, is discussed extensively by Zel'dovich and Novikov (1971) and the detailed observational consequences are examined by Shvartsman (1971) and by Shapiro (1973). Novikov and Thorne (1973) conclude that synchrotron radiation from such a black hole might be observable, but spherical accretion offers few good observational handles to probe detailed properties of the black hole. At least in some cases the radial flow of the gas is close to free fall.

In all cases the propagation of the electromagnetic radiation emitted by the matter is along null geodesics since all observable wavelengths are extremely small compared with the radius of the black hole.

Fortunately, as first shown by Carter (1968), the Hamilton-Jacobi equation for geodesics of the Kerr metric separates and the trajectories can be determined by quadratures. A complete set of constants of the motion for a test particle with momentum four-vector $p_{a}$ are $E=-p_{t}$, the energy, $L_{z}=p_{\phi}$, the angular momentum about the axis of symmetry, $\mu=\left(-p_{a} p^{a}\right)^{1 / 2}$, the rest mass, and finally the separation constant

$$
K=p_{\theta}^{2}+\left(L_{z}-a E \sin ^{2} \theta\right)^{2} / \sin ^{2} \theta+a^{2} \mu^{2} \cos ^{2} \theta .
$$

If $a=0$, so the black hole is spherically symmetric, $K$ is the square of the total angular momentum of the test particle. If $a \neq 0$ the total angular momentum is in general not a constant of the motion, and $K$ is not the total angular momentum even at infinity where the total angular momentum is constant.

Properties of timelike geodesics have been discussed by Ruffini and Wheeler (1970) and by Wilkins (1972), among others. Simplified formulas governing trajectories in the equatorial plane are given in Bardeen et al. (1972).

One immediate result of Equation (1) is that all orbits in the equatorial plane have

$$
Q \equiv K-\left(L_{z}-a E\right)^{2}=0 \text {. }
$$


Also, if $E=\mu, L_{z}=0$, and $Q=0$, the trajectories are 'radial' in the sense $\theta$ is constant for all values of $\theta$, though $\phi$ does vary. Such trajectories represent matter falling from rest at infinity with zero total angular momentum. As long as the flow is highly supersonic near the horizon calculations of 'spherical' accretion are not much more complicated in the Kerr geometry than they are in the spherically symmetric Schwarzschild geometry. However, a detailed calculation is necessary to see if there are any very striking observational consequences of the black hole's rotation in this case.

The rotation of the black hole does have important consequences for the case of disk accretion. If the angular momentum of the accreting material is in the same direction as the angular momentum of the black hole, the accretion disk will lie in the equatorial plane. The total gravitational energy released and the position of the inner edge of the accretion disk depend strongly on the value of $a / M$, particularly near $a / M=1$. The ratio $E / \mu$ for direct circular orbits in the equatorial plane of the black hole is plotted versus proper radial distance in Figure 1. The innermost stable

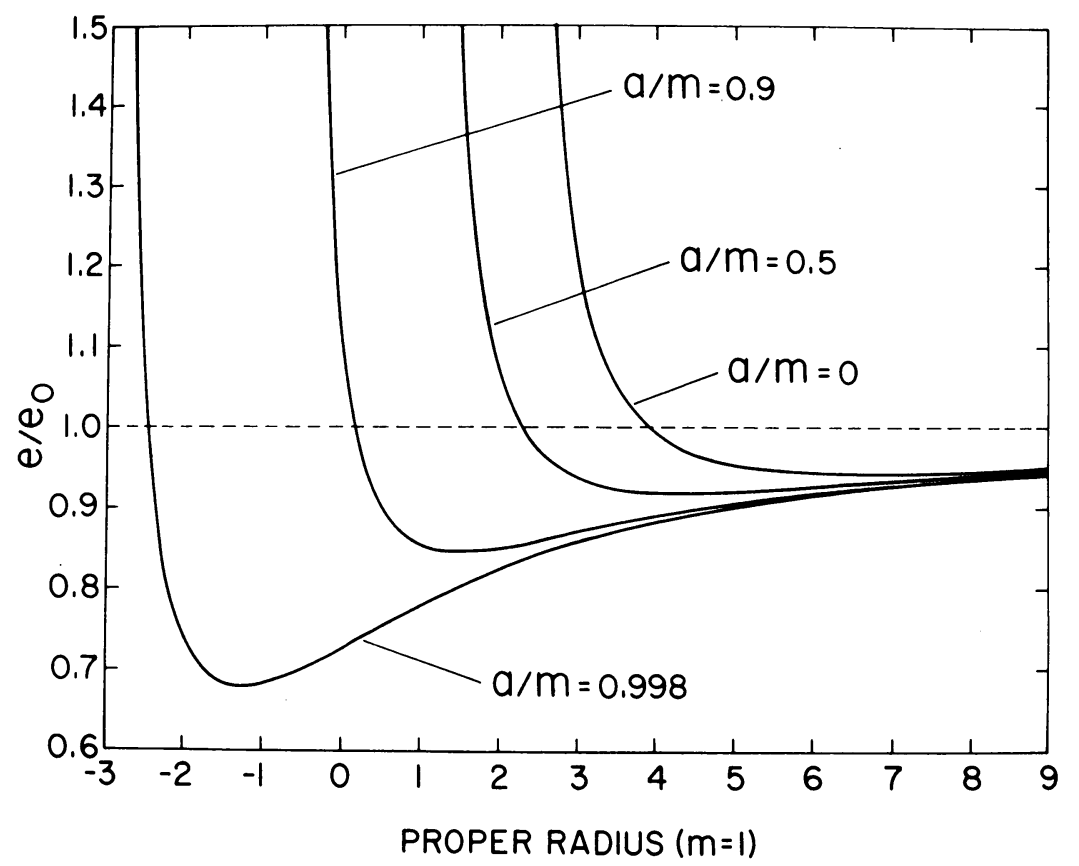

Fig. 1. The ratio of energy $e$ to rest mass energy $e_{o}$ as a function of proper radius for circular orbits in the equatorial plane of some Kerr black holes. In the text $e$ and $e_{o}$ are denoted by $E$ and $\mu$, respectively, and the mass $m$ of the black hole is denoted by $M$. When $a / m=c J / G M^{2}=1$ the ratio $e / e_{o}$ reaches a minimum value of 0.58 at a proper radius of minus infinity.

circular orbit is at the minimum of $E / \mu$ and represents the inner edge of the accretion disk in a simple model. The total gravitational energy release per unit rest mass in the accretion disk is $1-(E / \mu)_{\min }$. While an often quoted number is $1-(E / \mu)_{\min }=0.42$ for $a / M=1$, even for $a / M=0.998,1-(E / \mu)_{\min }$ is only 0.32 . When $a / M$ is close to one the ratio of proper radius interval to coordinate radius interval is large near 
the horizon. The innermost stable circular orbit is at coordinate radius $r \simeq M+$ $+[4(1-a / M)]^{1 / 3}$; the exact value for $a / M=0.998$ is $r / M=1.237$.

If some way could be found to calibrate the mass accretion rate independent of the luminosity of the accretion disk, the efficiency of energy release would be a sensitive test for the value of $a / M$.

When $a / M$ is close to one a sizable fraction of the gravitational energy release occurs inside the ergosphere, at $r<2 M$. However, this situation is not as favorable for observing the effects of the strong gravitational field as it might seem, since the turbulent viscosity in the disk transfers most of the energy out to somewhat larger radii before it is radiated (Novikov and Thorne, 1973).

Much of the hope for testing strong field predictions of general relativity lies in the possibility of observing local inhomogeneities or 'hot spots' in the inner part of the disk which would give rise to fluctuations in the X-ray output of the disk. Sunyaev (1972) has suggested that the periodic time-varying Doppler shift of the radiation emitted by such a hot spot would give a measure of the period of rotation of the disk. The period of rotation at the inner edge of the disk varies from $2 \pi(6)^{3 / 2} M$ for $a / M=0$ to $4 \pi M$ for $a / M=1$. If $M=10 M_{\odot}$ this is a variation in the minimum period from $5 \mathrm{~ms}$ to $0.6 \mathrm{~ms}$. The shortest time variations presently observable are roughly $100 \mathrm{~ms}$, but with bigger collecting areas on future X-ray satellites this may be reduced considerably. Since most of the energy flux comes from somewhat outside the inner edge of the disk, the dominant brightness variations might have periods several times larger than the numbers quoted above.

Other sources of fluctuations might be local oscillations of a region on the disk about its steady state. Some of these oscillations might be associated with the internal stresses in the disk, while others might be associated with perturbations in the orbits of the fluid elements. Some characteristic frequencies for the latter type of perturbation are plotted against coordinate radius, along with certain other physically important quantities, in Figure 2. The value of $a / M$ is 0.998 , but the main effect of changing $a / M$ is to change the radius of the inner edge of the disk, rather than change the values of the quantities at a fixed $r / M$.

The angular velocity of revolution as seen from infinity is $\Omega$. The quantities $K_{\|}$ and $K_{\perp}$ are epicyclic frequencies as measured by a comoving observer. If the circular geodesic orbit is perturbed, the perturbed orbit oscillates about the unperturbed orbit with the frequency $K_{\|}$if the perturbed orbit lies in the equatorial plane and with the frequency $K_{\perp}$ if the perturbation is perpendicular to the equatorial plane. Through the equation of geodesic deviation, $K_{\|}$is a measure of the tidal force acting in the radial direction in the comoving frame of the circular orbit, and $K_{\perp}$ is a measure of the tidal force acting in the $\theta$-direction in the comoving frame. By the axial symmetry, there is no such tidal force in the $\phi$-direction. The quantity $\bar{\Omega}$ is the locallymeasured angular velocity of rotation of the comoving frame, which is tied to the symmetry directions of the local (tidal) gravitational field, with respect to a nonrotating (Fermi-Walker-transported) frame. This angular velocity determines the centrifugal forces acting in the comoving frame. 
While $\Omega, \bar{\Omega}$, and $K_{\odot}$ increase roughly as $r^{-3 / 2}$ all the way in to the inner edge of the disk, $K_{\|}$, by definition, is zero at the marginally stable circular orbit. All four quantities are equal in the limit $r \gg M$. The local rate of shear of the disk, $\sigma$, is related to $K_{\|}$and $\Omega$ by

$$
K_{\|}=[2 \Omega(2 \Omega-\sigma)]^{1 / 2},
$$

and the vorticity, twice the angular velocity of a fluid element about its own center of mass, is $2 \Omega-\sigma$.

The velocity $v$ of the fluid element with respect to the zero-angular-momentum frame is more directly relevant to the appearance of the disk, since it gives the angle a zero angular momentum photon makes with the $\phi$-direction in the comoving frame.

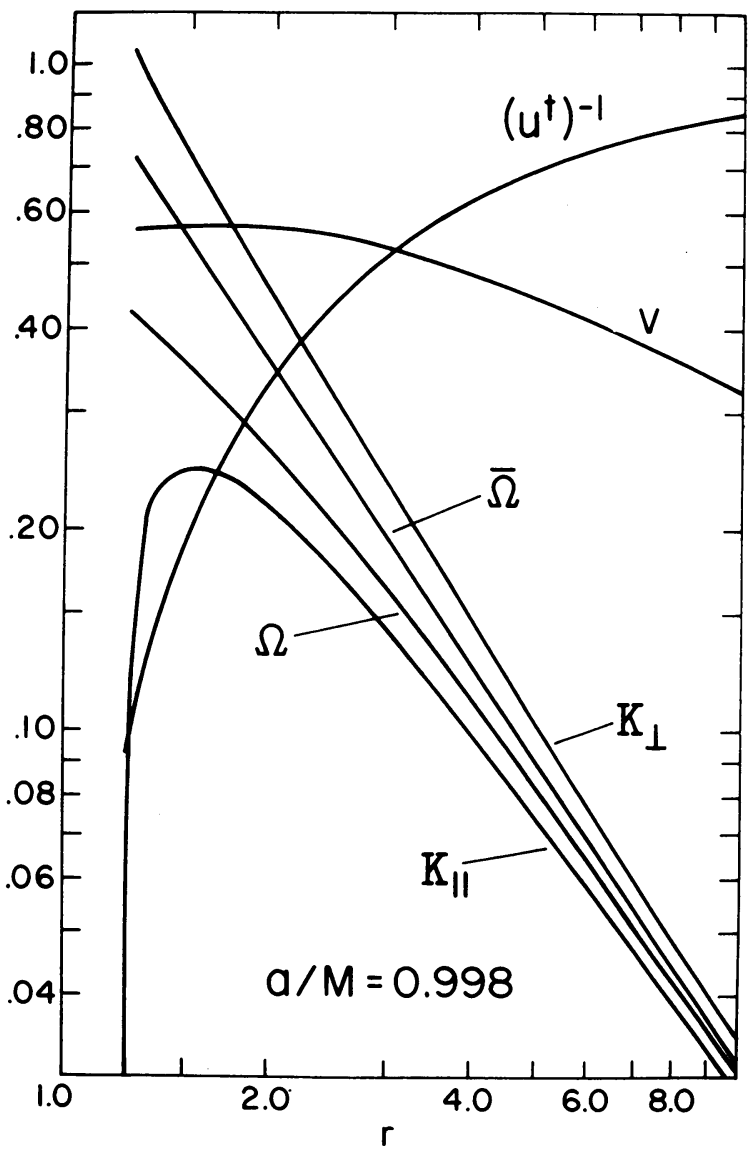

Fig. 2. Several physically important quantities characterizing circular orbits in the equatorial plane of a Kerr black hole with $a / M=0.998$ are plotted against coordinate radius $r$. The units are such that $c=G=M$ $=1$. The angular velocity of revolution of a test particle about the black hole as seen from infinity, $\Omega$, the angular velocity of rotation of the local tidal force field, $\bar{\Omega}$, the epicyclic frequencies in and perpendicular to the equatorial plane, $K_{\|}$and $K_{1}$, are important for understanding the dynamics of accretion disks. The quantity $\left(u^{t}\right)^{-1}$ is the zero-angular-momentum redshift factor and $v$ is the velocity of revolution with respect to a zero-angular-momentum observer. 
The local frequencies are redshifted by the factor $\left(u^{t}\right)^{-1}$ as seen from infinity on a time-averaged basis. However, the instantaneous redshift factor varies from a little less than $\left(u^{t}\right)^{-1}$ to (for a small fraction of the orbital period) roughly one if the observer is within about $30^{\circ}$ of the equatorial plane. Therefore, dynamic oscillations of a region on the disk are not completely frozen by the time dilation effect even if they take place near the inner edge of the disk for $a / M$ close to one. A locally periodic oscillation at $r / M$ close to one is not seen as a periodic oscillation from infinity. It comes alive for a fraction of the orbital period, when the local region is seen as relatively bright, and then tends to 'freeze' for the rest of the orbital period, when the local region is faint because its radiation suffers a large redshift. The very central part of a disk about an extreme Kerr hole will always be faint and time dilated when the observer is more than about $60^{\circ}$ from the equatorial plane.

To get a complete picture of the appearance of an accretion disk and to understand the transfer of energy by radiation in the region near the black hole one must look at the null geodesics in some detail. A null geodesic trajectory can be characterized by two non-trivial parameters, $\lambda=L_{z} / E$ and $\eta=Q^{1 / 2} / E$. These parameters can be related to the direction cosines of the beam of radiation in the frame comoving with the matter at the point of emission, on the one hand, and to the impact parameters which describe the position of the image seen by a distant observer, on the other hand. For an observer at a polar angle $\theta_{0}$ the impact parameter measuring the apparent perpendicular distance of the image from the symmetry axis is

$$
\alpha=-\lambda / \sin \theta_{0}
$$

and the impact parameter measuring the apparent perpendicular distance of the image from the projection of the equatorial plane is

$$
\beta=\left(\eta^{2}+a^{2} \cos ^{2} \theta_{0}-\lambda^{2} \cot ^{2} \theta_{0}\right)^{1 / 2} .
$$

If the matter at the point of emission is moving in a circular orbit in the equatorial plane of the black hole the ratio of frequency seen by a distant observer to frequency in the comoving frame at emission, the frequency shift factor $g$, depends on the angle the beam makes with the $+\phi$-direction, but is independent of the other direction cosines. In turn, the direction cosine with the $+\phi$-direction is independent of $\eta$.

Wilkins (1972) shows that a null geodesic can have at most one turning point. In the $(\eta, \lambda)$-plane the null geodesics with no turning points fill a compact region including the origin, and outside this region all the null geodesics have one turning point. The 'circular' null geodesic trajectories which stay forever at a constant value of $r$ are on the boundary of the no-turning-point region and are obviously all unstable. A parametric pair of equations for the boundary is

$$
\begin{aligned}
\lambda & =\frac{-r^{3}+3 M r^{2}-a^{2}(r+M)}{a(r-M)}, \\
\eta^{2} & =\frac{r^{3}}{a^{2}(r-M)^{2}}\left[4 a^{2} M-r(r-3 M)^{2}\right],
\end{aligned}
$$


and circular null trajectories are possible in the range of $r$ for which $\eta^{2}>0$ (Bardeen, 1973). A black hole in front of a bright source of much larger angular size, like a black hole in a binary system passing in front of a large bright star, looks like a black disk, with a radius of about $5 M$, whose boundary in the $(\alpha, \beta)$-plane corresponds to the locus of circular photon orbits in the $(\eta, \lambda)$-plane. Unfortunately, there seems to be no hope of observing this effect.

The appearance of a point source of radiation which radiates isotropically in its own rest frame as it moves in a circular geodesic orbit in the equatorial plane of an extreme $(a=M)$ Kerr black hole has been calculated by Cunningham and Bardeen (1973) for several orbital radii and for observers at several polar angles. There are an infinite number of images visible at any one time; for each image the energy flux is the product of its surface brightness, proportional to $[g(\lambda)]^{4}$, and its apparent angular size. Images can be classified by the number of times the beam of radiation crosses the equatorial plane between the source and observer. Images corresponding to more than one or two crossings are negligibly faint, but if the source orbits at $r \lesssim 3 M$ the one-crossing image is brighter on the average than the zero-crossing image. The time variations in the energy flux from the image seem to be primarily due to variations in surface brightness as $g(\lambda)$ varies; the variations are quite dramatic if the source is at $r \$ 20 M$ and the observer is within about $30^{\circ}$ of the equatorial plane.

These results would apply directly to something like a pulsar orbiting a massive black hole in the center of the Galaxy. An ordinary star wouldn't do, both because it would be torn apart by tidal forces unless the black hole were very massive $\left(M \gtrsim 10^{8}\right.$ $\left.M_{\odot}\right)$ and because its optical radiation would be absorbed by intervening dust. Unfortunately, pulsars are rare objects in the Galaxy, and while perhaps not completely inconceivable, it seems highly unlikely one would find itself sufficiently close to the hypothetical black hole.

If applied to accretion disks, the results do seem to confirm the possibility of large fluctuations in brightness from hot spots orbiting in the inner part of the disk. The beams of radiation which cross the equatorial plane will mostly be reabsorbed or scattered if $a / M$ is fairly close to one, which suggests that a good fraction of the radiation emitted inside $r \simeq 3 M$ will not escape directly to infinity. The whole energy balance of the innermost part of the disk will be substantially modified by the effect of the strong gravitational field on the propagation of the radiation. Also, the flaring of the disk far from the black hole (Shakura and Sunyaev, 1973) will be considerably enhanced over Newtonian estimates by the tendency of the gravitational field to focus radiation emitted by the inner part of the disk toward the equatorial plane. Cunningham (1973) has been exploring some of these possibilities.

If it happens that the orbital angular momentum of the binary system is not in the same direction as the spin angular momentum of the black hole, so the orbital plane of the accreting matter far from the black hole is tilted with respect to the equatorial plane of the black hole, the Lense-Thirring effect can have a big influence on the structure of the accretion disk even for $r \gg M$. If we take the polar axis to be along the axis of symmetry of the black hole, the Lense-Thirring effect causes the plane 
of a circular geodesic orbit at $r \gg M$ to precess with an angular velocity

$$
\mathrm{d} \phi / \mathrm{d} t \simeq 2 G J c^{-2} r^{-3}
$$

about the axis of symmetry of the black hole. In the case of an accretion disk the precession of the plane of the orbit of a fluid element is accompanied by a slow radial drift inward at a rate, in the middle region of Novikov and Thorne (1973),

$$
-v^{r} \sim r^{-2 / 5}
$$

Assuming a stationary flow, we get an unique precession angle at each radius,

$$
\phi_{p}(r)=2 G J c^{-2} \int_{\infty}^{r}\left(-v^{r}\right)^{-1} r^{-3} \mathrm{~d} r \sim r^{-8 / 5} .
$$

As $r$ decreases the accretion disk gets more and more twisted, in the mainer shown in Figure 3. The disk is sliced by the plane of the two angular momentum vectors. The scale of radius in Figure 3 depends on the coefficient of proportionality in Equation (9). For black holes in binaries $\varrho=1$ might correspond to about $1000 \mathrm{M}$. Typically, then, the twisting of the accretion disk becomes appreciable at a few hundred times the radius of the black hole, where the gravitational field is still Newtonian to a good approximation.

The picture drawn in Figure 3 neglects any torques due to viscosity. Crude estimates made by myself and $J$. Pettersen indicate that one component of the viscous torque tends to oppose the Lense-Thirring precession and another component tends to align the angular momentum of the orbiting material with that of the black hole. We expect that once the total precession angle has become moderately large the accretion disk will have relaxed into the equatorial plane of the black hole.

If the misalignment of the black hole with the orbital plane of the binary is fairly substantial, a sizable fraction of the energy released near the black hole may be redeposited in the disk in the region where the transition to the equatorial plane of the black hole occurs.

Whether or not misalignment can occur in binary systems like Cygnus X-1 is a complicated astrophysical question. Even if it is rather unlikely, the possibility of isolating an important relativistic effect warrants further examination.

\section{Energy Extraction From Black Holes}

Penrose (1969) was the first to suggest that rotating black holes can lose energy even though they accrete only matter and radiation with positive energy as measured by any local observer. Inside the ergosphere there exist physically realizable null and timelike geodesic trajectories with negative energy parameter $E$. When such a test particle falls into the black hole, the gravitational mass of the black hole decreases. The result is that the black hole has given up some 'rotational energy' to whatever injected the test particle into the negative energy orbit. 
Wheeler (1971) has suggested that if tidal forces disrupt a star in the ergosphere part of the material might find itself in such negative energy orbits, and the rest of the material might escape with more energy than the total rest mass energy of the original star. Mashoon (1972) has studied tidal disruption in the Kerr geometry without coming to any firm conclusions. However, Press (Bardeen et al., 1972)

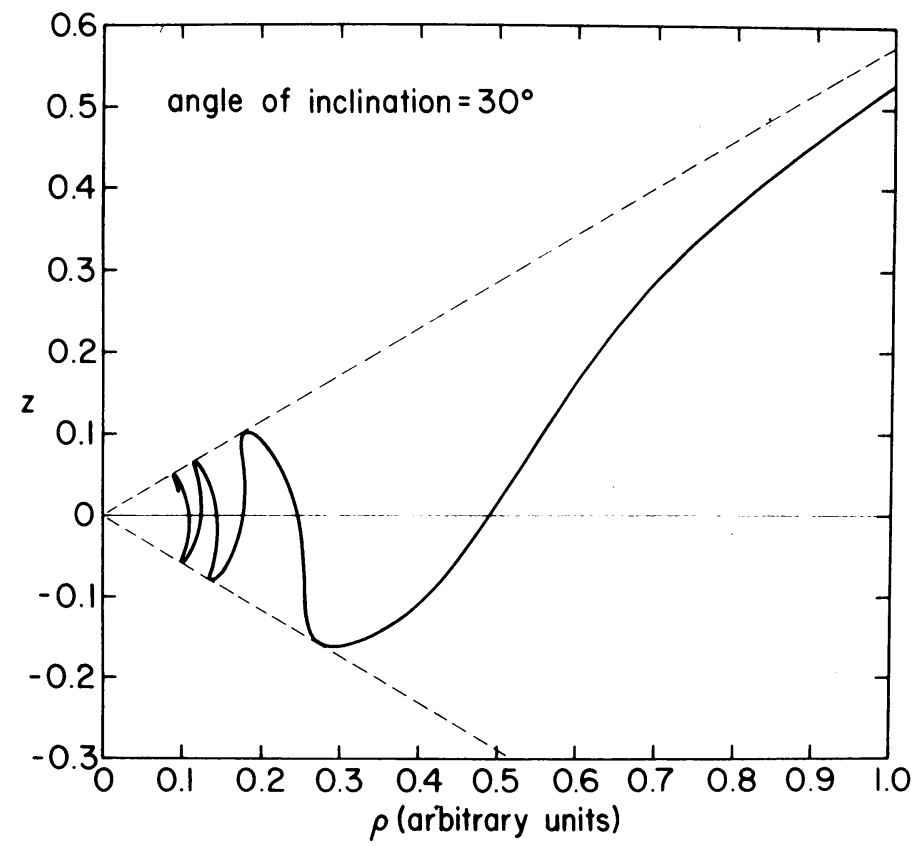

Fig. 3. A cross-section of an accretion disk which far from the black hole is tilted at an angle of $30^{\circ}$ with respect to the equatorial plane of the black hole. The units of the cylindrical coordinates $\varrho$ and $z$ are scaled to show the beginning of the twisting of the accretion disk by the Lense-Thirring dragging of inertial frames to best advantage. The tendency of the viscous forces to relax the accretion disk into the equatorial plane of the black hole is not taken into account; they are only used to calculate the radial velocity of the matter as in Novikov and Thorne (1973).

has shown that any negative energy orbit has a velocity of at least $0.5 c$ relative to any orbit which is accessible at $r \gg M$. It seems highly unlikely that tidal disruption can result in relative velocities much greater than the speed of sound in the original star, which rules out everything except neutron stars falling into small black holes.

Misner (1972) pointed out that waves can also extract energy from rotating black holes. Consider an electromagnetic or gravitational wave mode wich has frequency $\omega$ and axial angular eigenvalue $m$, so the wave amplitude is

$$
\psi \sim W(r, \theta) e^{i m \phi} e^{-i \omega t} .
$$

The relationship between a change in energy and a change in angular momentum of the black hole is

$$
\mathrm{d} M=\omega m^{-1} \mathrm{~d} J .
$$


On the other hand, $\mathrm{d} M, \mathrm{~d} J$, and the change in area $\mathrm{d} A$ are related by (Hartle and Hawking, 1972)

$$
\mathrm{d} M=(8 \pi)^{-1} \kappa \mathrm{d} A+\Omega_{H} \mathrm{~d} J,
$$

where $\kappa$ is the 'surface gravity' of the black hole and $\Omega_{H}$ is the angular velocity of the horizon (see Bardeen et al., 1973). Combining Equations (12) and (13) gives

$$
\mathrm{d} M=(8 \pi)^{-1} \omega\left(\omega-m \Omega_{H}\right)^{-1} \kappa \mathrm{d} A .
$$

Since the area of the black hole can only increase (Hawking, 1971), if

$$
0<\omega<m \Omega_{H},
$$

the black hole mass decreases. If such a mode scatters off a rotating black hole the outgoing energy flux is greater than the ingoing energy flux, hence superradiant scattering. Numerical calculations by Teukolsky and Press (Teukolsky, 1973) indicate that the superradiance is less than $4 \%$ for electromagnetic waves, though Starobinsky and Churilov (1973) have shown analytically it can be as much as $137 \%$ for gravitational waves. No very plausible mechanism has been proposed for superradiance as such manifesting itself in a noticeable way in astrophysics (but see Press and Teukolsky (1972) on the 'black hole bomb').

Perhaps the astrophysically most interesting possibility is the existence of 'floating orbits' (Press and Teukolsky, 1972). From Equation (14) an orbiting test particle that has an angular velocity $\Omega<\Omega_{H}$ will extract energy from the black hole. If the energy extracted from the black hole exceeds the energy radiated to infinity, instead of spiralling into the black hole the particle will spiral outward until there is a balance; the particle then 'floats' at constant radius as it acts as a catalyst for extracting energy from the black hole. The calculations to check this possibility for electromagnetic or gravitational waves have not yet been done, though the mathematical techniques are now at hand, thanks to Teukolsky (1972).

\section{Conclusion}

My discussion has focussed on what relativistic effects may have observable consequences for models of accretion disks around black holes in binary systems, since at the present time there are the only systems in which there seems to be positive evidence for the existence of black holes. Black holes with accretion disks may also be the source of some of the non-thermal activity in galactic nuclei, from the modest level in the Galaxy to the most powerful quasars. Particularly for quasars, and probably in some X-ray binaries, the accretion rate may approach or even exceed the Eddington limit. The thin disk approximation, in which the fluid elements move in nearly geodesic trajectories in the equatorial plane, breaks down near the black hole when the luminosity approaches the Eddington limit. What then happens has not been explored in any detail, though Shakura and Sunyaev (1973) have given a qualitative picture. Particularly if the black hole is rapidly rotating any quantitative analysis will have to take into account strong relativistic effects. 
Black holes also provide a number of mechanisms for generating gravitational waves, some of which are certainly of astrophysical interest. Such mechanisms are probably the only hope for explaining Weber's graviational wave experiments, if this is possible at all with conventional general relativity.

Through my whole discussion I have implicitly assumed that Kerr black holes are stable. Numerical calculations of Press and Teukolsky (1973) indicate rather strongly that the Kerr metric is stable to gravitational and electromagnetic perturbations, but a completely airtight proof of stability is not yet at hand.

What I hope my talk illustrates is that general relativity is more than a background in which to do astrophysics; rather, the relativistic aspects of black holes can generate qualitatively new observational effects. Much more theoretical work is necessary to develop the detailed models which will hopefully make possible a new type of confrontation of general relativity theory with observation.

\section{References}

Bardeen, J. M.: 1970, Nature 226, 64.

Bardeen, J. M.: 1973, in C. DeWitt and B. DeWitt (eds.), Black Holes, Gordon and Breach, New York, London, Paris, p. 229.

Bardeen, J. M., Carter, B., and Hawking, S. W.: 1973, Commun. Math. Phys. 31, 161.

Bardeen, J. M., Press, W. H., and Teukolsky, S. A. : 1972, Astrophys. J. 178, 347.

Bardeen, J. M. and Wagoner, R. V.: 1971, Astrophys. J. 167, 359.

Carter, B.: 1968, Phys. Rev. 174, 1558.

Carter, B.: 1971, Phys. Rev. Letters 26, 331.

Cunningham, C. T. and Bardeen, J. M.: 1973, Astrophys. J. 183, 237.

Cunningham, C. T.: 1973, Ph.D. Thesis, University of Washington.

Hartle, J. B. and Hawking, S. W.: 1972, Commun. Math. Phys. 26, 87.

Hawking, S. W.: 1971, Phys. Rev. Letters 26, 1344.

Hawking, S. W. and Ellis, C. F. R. : 1973, The Large Scale Structure of Space-Time, Cambridge University Press, Cambridge.

Israel, W.: 1967, Phys. Rev. 164, 1776.

Kerr, R. P.: 1963, Phys. Rev. Letters 11, 238.

Lynden-Bell, D.: 1969, Nature 223, 690.

Lynden-Bell, D. and Rees, M. J.: 1971, Monthly Notices Roy. Astron. Soc. 152, 461.

Mashoon, B.: 1972, Ph.D. Thesis, Princeton University.

Misner, C. W.: 1972, Phys. Rev. Letters 28, 994.

Newman, E. T., Couch, E., Chinnapared, R., Exton, A., Prakash, A., and Torrence, R.: 1965, J. Math. Phys. 6, 918.

Novikov, I. D. and Thorne, K. S.: 1973, in C. DeWitt and B. DeWitt (ed.), Black Holes, Gordon and Breach, New York, London, Paris.

Peebles, P. J. E.: 1972, Gen. Rel. Grav. 3, 63.

Press, W. H. and Teukolsky, S. A.: 1972, Nature 238, 211.

Press, W. H. and Teukolsky, S. A.: 1973, Astrophys J. 185, 649.

Pringle, J. E. and Rees, M. J.: 1972, Astron. Astrophys. 21, 1.

Ruffini, R. and Wheeler, J. A.: 1970, in A. F. Moore and V. Hardy (ed), The significance of Space Research for Fundamental Physics, European Space Research Organization, Paris.

Shakura, N. I. and Sunyaev, R. A.: 1973, Astron. Astrophys. 24, 337.

Shapiro, S. L.: 1973, Astrophys. J. 180, 531.

Shvartsman, V. F.: 1971, Soviet Astron. AJ 15, 277.

Starobinski, A. A. and Churilov, S. M.: 1973, Zh. Eksperim. Teor. Fiz. 65, 3.

Sunyaev, R. A.: 1972, Astron. Zh. 49, 1153.

Teukolsky, S. A.: 1972, Phys. Rev. Letters 29, 1114. 
Teukolsky, S. A.: 1973, Ph.D. Thesis, California Institute of Technology.

Thorne, K. S.: 1974, Astrophys. J., to be published.

Wheeler, J. A.: 1971, in D. J. K. O,Connell (ed.), Nuclei of Galaxies, North-Holland Publishing Co., Amsterdam, London.

Wilkins, D. C.: 1972, Phys. Rev. D5, 814.

Zel'dovich, Ya. B. and Novikov, I. D.: 1971, Relativistic Astrophysics, Vol. I, University of Chicago Press, Chicago. 